\title{
RESEARCH
}

Open Access

\section{Effect of lipid metabolism disorder on liver function in patients with malignant tumors after chemotherapy: a case-control study}

Yan Sun ${ }^{1+}$, Nie Zhang ${ }^{2+}$, Yun-Long Ding ${ }^{3+}$, Li-Jiang Yu', Jun Cai ${ }^{1}$, De Ma' ${ }^{1}$, Wu Yang ${ }^{1}$, Wang-Kun Lu ${ }^{1}$ and Jia-Li Niu ${ }^{4^{*}}$ (D)

\begin{abstract}
Background: This study aims to investigate the effect of lipid metabolism disorder on liver function in patients with malignant tumors after chemotherapy.

Method: A total of 428 patients with malignant tumors with normal liver function in our hospital between May 2013 to June 2018 were divided into an observation group (lipid metabolism disorder, $n=265$ ) and control group (normal lipid metabolism, $n=163$ ). The lipid metabolism levels and liver damage of the two groups were compared before and after chemotherapy.

Results: No significant differences in age, gender, body mass index, tumor types, history of surgery, levels of alanine aminotransferase (ALT; an indicator of liver function), and chemotherapy regimen were observed between the two groups. However, the observation group showed increased levels of total cholesterol $(P=0.000)$, triglycerides $(P=0.000)$, and low-density lipoprotein $(P=0.01)$, as well as decreased levels of high-density lipoprotein $(P=0.000)$ before chemotherapy compared with the control group. Furthermore, patients with lipid metabolism disorders were more likely to develop abnormal liver function after chemotherapy. Moreover, mixed lipid metabolism disorder was more likely to cause severe liver damage after chemotherapy. Additionally, the number of patients with lipid metabolism disorders after chemotherapy $(n=367)$ was significantly increased compared with before chemotherapy $(n=265)(P<0.01)$, indicating that chemotherapy might induce or aggravate an abnormal lipid metabolism.
\end{abstract}

Conclusions: After receiving chemotherapy, patients with malignant tumors presenting lipid metabolism disorders are more prone to liver damage and lipid metabolism disorders than patients with a normal lipid metabolism.

Keywords: Lipid metabolism, Tumor, Chemotherapy, Liver function

\footnotetext{
* Correspondence: jialiniu@aliyun.com

†Yan Sun, Nie Zhang, and Yun-Long Ding are contributed equally to this work.

†Yan Sun, Nie Zhang, and Yun-Long Ding should be considered co-first authors.

${ }^{4}$ Department of Clinical Pharmacy, Jing Jiang People's Hospital, the Seventh

Affiliated Hospital of Yangzhou University, No. 28, Zhongzhou Road,

Jingjiang, Jiangsu 214500, China

Full list of author information is available at the end of the article
}

(c) The Author(s). 2019 Open Access This article is distributed under the terms of the Creative Commons Attribution 4.0 International License (http://creativecommons.org/licenses/by/4.0/), which permits unrestricted use, distribution, and reproduction in any medium, provided you give appropriate credit to the original author(s) and the source, provide a link to the Creative Commons license, and indicate if changes were made. The Creative Commons Public Domain Dedication waiver (http://creativecommons.org/publicdomain/zero/1.0/) applies to the data made available in this article, unless otherwise stated. 


\section{Background}

Malignant tumors are a serious threat to human health, and chemotherapy remains one of the main treatments. However, chemotherapy has many toxic side effects [1]. For instance, as most chemotherapeutic drugs are metabolized by the liver, liver toxicity is one of the most common side effects [2]. Clinical manifestations of chemotherapy-induced liver damage are diverse, ranging from slight liver function abnormalities to severe cases of toxic hepatitis or fulminant hepatic failure. Hepatic toxicity is generally reversible [3], but may still cause fibrosis or cirrhosis if discontinued. In addition, liver damage caused by chemotherapeutic drugs may lead to lipid metabolism disorders and even hyperlipidemia $[4,5]$.

High-fat, high-energy diets have resulted in an increase in the incidence of lipid metabolism disorders [6]. Under this condition, reduced transport of fat causes lipids to accumulate in liver cells, resulting in a fatty liver [7]. Fat accumulation in the liver can affect the function of or destroy liver cells and cause connective tissue hyperplasia and cirrhosis, which affects drug metabolism [8]. Because chemotherapeutic drugs can cause liver damage and liver damage is associated with lipid metabolism disorders, we suspect that lipid metabolism disorders may be related to liver damage caused by chemotherapeutic drugs, but no research on this topic has been reported.

In the present study, we examined lipid metabolism in patients with malignant tumors who were treated at our hospital in recent years and assessed impairments in liver function after chemotherapy to investigate the effect of lipid metabolism disorders on liver function in patients undergoing chemotherapy.

\section{Materials and methods}

\section{General information}

This study included patients who were undergoing chemotherapy at our hospital from May 2013 to June 2018. Exclusion criteria were patients with diseases associated with liver damage, such as liver metastasis, viral hepatitis, drug-induced liver disease, total parenteral nutrition, and hepatolenticular degeneration. Inclusion criteria were patients diagnosed with malignant tumors via a pathological or cytological examination who required chemotherapy, had no chemotherapy contraindications, were aged $\geq 18$ years, had normal liver function (alanine aminotransferase (ALT) level $\leq 50 \mathrm{IU} / \mathrm{L}$ ), and had a Kamofsky performance status $\geq 70$ points. Before chemotherapy, tests of blood lipids and liver function were performed on all patients.

\section{Procedure}

The blood lipid levels and liver function of each patient were assessed before chemotherapy. All indicators were in the normal range before chemotherapy. All patients underwent chemotherapy with a combination regimen according to the pathological type of malignancy. Common chemotherapy drugs included oxaliplatin, 5-fluorouracil, paclitaxel, cyclophosphamide, epirubicin, cisplatin, platinum, vinorelbine, gemcitabine, capecitabine, vincristine, and irinotecan, among others. Blood lipid levels and liver function were also measured approximately 1 week after chemotherapy was initiated. If abnormal liver function was observed during chemotherapy, chemotherapy was suspended and relevant drugs were administered as a protective treatment. Liver function was reassessed every 5-7 days until it returned to normal levels, after which chemotherapy was continued. After chemotherapy, liver function and blood lipid levels were analyzed again. In this study, liver function and blood lipid levels were all measured approximately 1 week after chemotherapy was initiated. Venous blood was collected in the morning from all patients who had fasted for more than $8 \mathrm{~h}$, and total cholesterol (TC), triglyceride (TG), low-density lipoprotein cholesterol (LDL-C), and high-density lipoprotein cholesterol (HDL-C) levels were determined. Lipid indicators, liver function and ALT levels were assessed using enzymatic methods.

\section{Types of lipid metabolism disorders}

Based on the recommendations for the prevention and treatment of dyslipidemia, lipid metabolism disorders are classified as follows [9]: 1. hypercholesterolemia (fasting serum TC level exceeding $5.72 \mathrm{mmol} / \mathrm{L}$ ); 2 . hypertriglyceridemia (fasting serum TG level exceeding $1.70 \mathrm{mmol} / \mathrm{L}$ ); 3. mixed hyperlipidemia (increased fasting serum TC and TG levels); and 4. low high-density lipoproteinemia (fasting serum HDL level $<0.9 \mathrm{mmol} / \mathrm{L}$ ).

\section{Criteria for evaluating liver function}

According to the World Health Organization standards for the toxicity and side effects of anticancer drugs, the evaluation of liver function was defined using the following criteria: 1 . normal liver function, serum ALT level $\leq$ $1.25 \mathrm{~N}(N=40 \mathrm{IU} / \mathrm{L}) ; 2$. liver function damage I level, 1.25 $\mathrm{N}<$ serum ALT level $<2.5 \mathrm{~N} ; 3$. liver function damage II level, $2.5 \mathrm{~N}<$ serum ALT level $<5 \mathrm{~N}$; 4 . liver function damage III level, $5 \mathrm{~N}<$ serum ALT level $<10 \mathrm{~N}$; and 5 . liver function damage IV level, serum ALT level $>10 \mathrm{~N}$.

\section{Non-alcoholic fatty liver disease (NAFLD)}

According to the guidelines for the diagnosis and treatment of NAFLD [10], the disease is divided into non-alcoholic simple fatty liver disease, non-alcoholic fatty liver hepatitis and non-alcoholic fatty liver cirrhosis. 


\section{Statistical analysis}

Statistical analyses were performed using SPSS 20.0 statistical analysis software. The count data are presented as means \pm standard deviations. T-tests were used for comparisons between groups. Dichotomous variables were analyzed using the $\chi^{2}$ test. If the number of observations was less than 5, Fisher's exact probability test was used to calculate the $P$ value. $P<0.05$ was considered statistically significant.

\section{Results}

The observation group showed increased levels of TC, TG, and LDL and decreased levels of HDL before chemotherapy

A total of 428 patients with malignant tumors with normal liver function (199 males and 229 females, with an average age of $62.4 \pm 9.2$ years) were enrolled in our hospital from May 2013 to June 2018. These patients were divided into the lipid metabolism disorder group (observation group, $n=265$ ) and normal lipid metabolism group (control group, $n=163$ ) according to the lipid metabolism status before chemotherapy. In the observation group, 69 patients (26.0\%) had high cholesterol levels, 98 patients $(37.0 \%)$ had high TG levels, 61 patients $(23.0 \%)$ had a mixed type, and 37 patients $(14.0 \%)$ had a low HDL-C type. General characteristics of the two groups are shown in Table 1. No significant differences in age, gender, body mass index (BMI), tumor types, and history of surgery between the two groups.
Furthermore, significant differences in ALT levels (a sensitive indicator of liver function) before chemotherapy were not observed between the observation (26.2 \pm $7.8 \mathrm{IU} / \mathrm{L})$ and control groups $(25.4 \pm 9.4 \mathrm{IU} / \mathrm{L})$. However, the observation group showed increased levels of TC $(P$ $=0.000)$, TG $(P=0.000)$, and LDL $(P=0.01)$ and decreased levels of HDL $(P=0.000)$ before chemotherapy compared with the control group. Moreover, significant differences in the chemotherapy regimen were not observed between the two groups (Table 2).

\section{The observation group showed a higher incidence of liver damage after chemotherapy}

The levels of liver damage after chemotherapy in the two groups are shown in Table 3. The incidence of liver damage after chemotherapy was $47.2 \%$ in the observation group (125/265), with 111 patients presenting level I damage (41.9\%), 8 patients presenting level II damage (3.0\%), and 6 patients presenting level III damage (2.3\%), whereas the incidence in the control group was $20.9 \%$ (34/163), with 29 presenting level I damage (17.8\%), 3 patients presenting level II damage (1.8\%), and 2 patients presenting level III damage (1.2\%). The incidence of liver damage after chemotherapy in the observation group was significantly greater than in the control group $(P<$ 0.01). Based on these results, patients with lipid metabolism disorder are more prone to liver damage after chemotherapy than patients with normal lipid metabolism.

Table 1 Comparison of the general conditions between the two groups $[\bar{x} \pm \mathrm{s} /$ case $(\%)]$

\begin{tabular}{llll}
\hline Item & Observation $(n=265)$ & Control $(n=163)$ & $P$ \\
\hline Age & $62.0 \pm 9.3$ & $61.3 \pm 9.0$ & $76(46.6)$ \\
Male & $123(46.4)$ & $21.8 \pm 2.6$ & 0.247 \\
BMI & $22.2 \pm 2.0$ & & 0.996 \\
Tumor types & & $61(37.4)$ & 0.086 \\
Gastrointestinal cancer & $105(39.6)$ & $37(22.7)$ & 0.650 \\
Lung cancer & $47(17.7)$ & $32(19.6)$ & 0.209 \\
Breast cancer & $56(21.1)$ & $16(9.8)$ & 0.709 \\
Gynecologic cancer & $25(9.4)$ & $6(3.7)$ & 0.896 \\
Malignant lymphoma & $10(3.8)$ & $11(6.7)$ & 0.961 \\
Other tumors & $22(8.3)$ & $116(71.2)$ & 0.559 \\
Surgery & $182(68.7)$ & $25.4 \pm 9.4$ & 0.587 \\
ALT before chemotherapy & $26.2 \pm 7.8$ & $4.7 \pm 0.7$ & 0.361 \\
TC before chemotherapy & $5.5 \pm 1.2$ & $1.3 \pm 0.2$ & 0.000 \\
TG before chemotherapy & $1.8 \pm 0.6$ & $1.4 \pm 0.4$ & 0.000 \\
HDL before chemotherapy & $1.3 \pm 0.5$ & $2.9 \pm 0.8$ & 0.000 \\
LDL before chemotherapy & $3.2 \pm 1.1$ & 0.010 \\
\hline BMI body mass index, ALT & &
\end{tabular}

$B M I$ body mass index, $A L T$ alanine aminotransferase, $T C$ total cholesterol, $T G$ triglycerides, $H D L$ high-density lipid, $L D L$ low-density lipoprotein; $P<0.05$ was considered statistically significant 
Table 2 Comparison of the chemotherapy regimens between the two groups [case(\%)]

\begin{tabular}{llll}
\hline Item & Observation $(n=265)$ & Control $(n=163)$ & $P$ \\
\hline CHOP & $10(3.8)$ & $6(3.7)$ & 0.961 \\
CP & $4(1.5)$ & $0(0)$ & 0.302 \\
DP & $2(0.8)$ & $3(1.8)$ & 0.374 \\
DVP & $0(0)$ & $1(0.6)$ & 0.381 \\
FOLFIRI & $14(5.3)$ & $7(4.3)$ & 0.646 \\
FOLFOX & $76(28.7)$ & $39(23.9)$ & 0.281 \\
GP & $26(9.8)$ & $19(11.7)$ & 0.546 \\
IP & $2(0.8)$ & $0(0)$ & 0.527 \\
NF & $11(4.2)$ & $6(3.7)$ & 0.809 \\
NP & $39(14.7)$ & $30(18.4)$ & 0.314 \\
PVB & $0(0)$ & $2(1.2)$ & 0.144 \\
PVD & $2(0.8)$ & $2(1.2)$ & 0.637 \\
TA & $25(9.4)$ & $13(8)$ & 0.606 \\
TF & $6(2.3)$ & $9(5.5)$ & 0.075 \\
TOX & $2(0.8)$ & $0(0)$ & 0.527 \\
TP & $44(16.6)$ & $24(14.7)$ & 0.605 \\
VA & $0(0)$ & $2(1.2)$ & 0.144 \\
VP & $2(0.8)$ & $0(0)$ & 0.527 \\
\hline
\end{tabular}

Types of lipid metabolism disorders before chemotherapy correlated with liver function after chemotherapy

The correlations between the types of lipid metabolism disorders before chemotherapy and liver function after chemotherapy are presented in Table 4. No significant differences in the percentages of patients with normal liver function and liver damage at levels I and level II were observed among the hypercholesterolemia group, hypertriglyceridemia group, the mixed hyperlipidemia group, and low-HDL group. However, liver function at damage level III after chemotherapy was observed in 6 patients in the mixed hyperlipidemia group, but was not observed in the other groups. Moreover, a significant difference in the percentage of patients presenting function damage level III after chemotherapy was observed among the four groups $(P=0.000)$. In addition, a statistically significant difference in the degree of liver damage after chemotherapy was observed among the four groups $(P=0.002)$. Thus, a significant correlation exists between the type of lipid metabolism disorder before chemotherapy and liver function after chemotherapy.

\section{Changes in lipid metabolism before and after chemotherapy}

In addition to investigating the effect of lipid metabolism disorders on damage to liver function in patients with cancer after chemotherapy, we also evaluated the effect of chemotherapy on lipid metabolism in patients. Two hundred sixty-five patients presented with dyslipidemia and 163 patients presented with normal lipid metabolism before chemotherapy. Interestingly, the number of patients with dyslipidemia $(n=367)$ after chemotherapy was significantly increased compared with before chemotherapy $(P<0.01)$ (Table 5). Based on these data, chemotherapy might induce or aggravate lipid metabolism disorders.

\section{Discussion}

Clinically, many patients receiving chemotherapy develop lipid metabolism disorders. In addition, the administration of chemotherapy to patients with lipid metabolism disorders may increase the risk of liver damage. However, currently, evidence for the relationship between lipid metabolism disorders and liver damage after chemotherapy is limited. The results from the present study showed increased levels of TC, TG, and LDL and decreased levels of HDL in the patients with lipid metabolism disorder before chemotherapy compared with the patients with normal lipid metabolism (Table 1). Furthermore, patients with lipid metabolism disorders are more likely to develop abnormal liver function after chemotherapy (Table 3). Moreover, mixed lipid metabolism disorder is more likely to cause severe liver damage after chemotherapy (Table 4). In addition, the number of patients with lipid metabolism disorders after chemotherapy $(n=367)$ was significantly increased compared with before chemotherapy $(n=265)$ (Table 5), indicating that chemotherapy potentially induces or aggravates abnormal lipid metabolism.

According to the evidence, chemotherapy drugs can cause lipid metabolism disorders. Paclitaxel and platinum-based chemotherapy caused transient dyslipidemia in three patients with cancer [11]. The chemotherapy drug 5 -fluorouracil induces steatosis by generating reactive oxygen species [12]. Lipid metabolism disorders may be induced by chemotherapy due to drug toxicity [13]. Consistent with this finding, chemotherapy induced or aggravated abnormal lipid metabolism in the present study, as indicated by the increased number of patients with lipid metabolism disorders after chemotherapy.

Table 3 Comparison of liver function damage after chemotherapy between the two groups [case(\%)]

\begin{tabular}{llllll}
\hline Group & Level I & Level II & Level III & Level IV & Total \\
\hline Observation $(n=265)$ & $111(41.9)$ & $8(3.0)$ & $6(2.3)$ & $0(0)$ & $125(47.2)^{* *}$ \\
Control $(n=163)$ & $29(17.8)$ & $3(1.8)$ & $2(1.2)$ & $0(0)$ & $34(20.9)$ \\
\hline
\end{tabular}

Compared with the control group, ${ }^{*} P<0.05,{ }^{* *} P<0.01$ 
Table 4 Types of lipid metabolism disorders and liver function after chemotherapy [case (\%)]

\begin{tabular}{|c|c|c|c|c|c|}
\hline Type & Hypercholesterolemia & Hypertriglyceridemia & Mixed & Low-HDL & $P$ \\
\hline Normal & $39(56.5)$ & $48(49.0)$ & $30(49.2)$ & $23(62.2)$ & 0.461 \\
\hline Level I & $30(43.5)$ & $48(49.0)$ & $21(34.4)$ & $12(32.4)$ & 0.183 \\
\hline Level II & $0(0)$ & $2(2.0)$ & $4(6.6)$ & $2(5.4)$ & 0.067 \\
\hline Level III & $0(0)$ & $0(0)$ & $6(9.8)$ & $0(0)$ & 0.000 \\
\hline Total & 69 & 98 & 61 & 37 & 0.002 \\
\hline
\end{tabular}

Chemotherapeutic drugs have been shown to induce liver damage [12, 14, 15]. Almost all chemotherapeutic drugs are metabolized by the liver, the metabolic center of the body, and thus easily lead to drug-induced liver damage. Chemotherapeutic drugs mainly cause liver damage mainly through the following three pathways: (1) drugs and their metabolites directly damage liver cells; (2) chemotherapy may aggravate liver damage in patients with pre-existing liver diseases; and (3) pre-existing liver diseases might reduce the activity of drug-metabolizing enzymes and prolong the duration of drug action, thereby increasing the toxicity of chemotherapeutic drugs.

As shown in recent studies, lipid metabolism is closely related to the occurrence and development of tumors. Patients with tumors tend to have lipid metabolism disorders. In the present study, compared with patients with normal lipid metabolism, patients with malignant tumors presenting dyslipidemia showed an increased incidence of hepatic impairment after chemotherapy. Thus, hepatotoxicity should be more carefully assessed when chemotherapeutic drugs are administered to patients with lipid metabolism disorders.

The mechanisms by which lipid metabolism disorders affect liver function after chemotherapy are not yet completely understood, but may be related to the factors described below. (1) Excess lipids directly exert adverse effects on liver function. When a lipid metabolism disorder occurs, the liver is unable to transport fat in a timely manner, thus causing fat to accumulate in liver cells and resulting in fatty liver. Fat accumulation in the liver affects liver function and destroys liver cells, subsequently reducing the liver compensatory capacity and causing connective tissue hyperplasia, cirrhosis, and liver damage [16]. (2) Pre-existing dyslipidemia indirectly

Table 5 Changes in lipid metabolism before and after chemotherapy

\begin{tabular}{llll}
\hline $\begin{array}{lll}\text { Lipid } \\
\text { metabolism } \\
\text { before } \\
\text { chemotherapy }\end{array}$ & Lipid metabolism after chemotherapy & Total \\
\cline { 2 - 3 } & Disorder & Normal & \\
\hline Disorders & 239 & 26 & 265 \\
Normal & 128 & 35 & 163 \\
Total & 367 & 61 & 428 \\
\hline
\end{tabular}

contributes to liver damage after chemotherapy. Most chemotherapeutic drugs are cytotoxic and immunosuppressive agents, causing a certain degree of damage to the liver themselves [17]. Chronic liver damage is present in patients with lipid metabolism disorders, resulting in a decreased drug metabolism capacity and aggravation of liver damage. (3) Pro-inflammatory cytokines exacerbate liver damage. For example, tumor cell necrosis after chemotherapy causes the release of tumor necrosis factor, one of the main factors involved in liver cell damage and even hepatic necrosis [18]. (4) The oxidative stress response also contributes to liver damage. Excess intake of free fatty acids causes oxidative overload in the mitochondria of hepatocytes, producing more reactive oxygen species (ROS). The expression of uncoupling protein 2 is compensatorily increased to reduce ROS production, and a large amount of adenosine triphosphate is consumed, leading to the apoptosis or even necrosis of hepatocytes [19]. Hepatocyte necrosis produces a large amount of inflammatory factors, further increasing the production of free radicals.

Chemotherapy-induced liver damage in patients with cancer may occur during chemotherapy or after the total course. Although liver function impairment may be reversed in some patients after treatment, abnormal liver function may lead to an interruption of anti-tumor therapy and jeopardize the prognosis of patients [20]. The clinical consequences of liver damage after chemotherapy in patients with cancer vary. Mild cases may only produce an asymptomatic increase in ALT levels, spontaneous reversal may occur in some patients, and severe cases may involve jaundice, ascites, coagulation abnormalities, encephalopathy and other signs of liver failure, with a high mortality rate if not treated effectively [21]. The symptoms experienced by these patients are mild, with most having no symptoms or signs, and abnormalities are only observed when liver function is measured. For patients with symptoms and signs, except for jaundice, the main symptoms are fatigue, anorexia, bloating, abdominal pain, and fever. The clinical manifestations of patients are similar to the original disease; they are not specific and are difficult to identify. Thus, the clinical manifestations of liver damage after chemotherapy are subtle in some patients and may not be detected in a timely manner, with a risk of worsening adverse 
reactions during continued treatment. Therefore, transaminase levels should be regularly monitored in patients with cancer who are undergoing chemotherapy. Once abnormal liver function is detected, a hepatoprotective enzyme treatment should be administered in a timely manner to prevent the further aggravation of the disease and ensure the successful completion of chemotherapy.

Lipid metabolism disorders are major risk factors for cardiovascular and cerebrovascular diseases and exert important effects on the metastasis and prognosis of some tumors, particularly breast and gastrointestinal tumors. Therefore, blood lipid levels must be controlled in patients with tumors. Patients with cancer should pay special attention to their eating habits and avoid high-fat diets to prevent abnormal lipid metabolism. Routine screening of blood lipid and transaminase levels before chemotherapy is recommended for patients with cancer, as are preventive measures to regulate lipid metabolism and protect liver function to reduce the incidence of hepatic damage, liver failure and tumor-related mortality. Improvements in the quality of life of patients with cancer will have a positive impact on the prognosis.

\section{Conclusions}

In conclusion, patients with malignant tumors presenting lipid metabolism disorders are more prone to liver damage after chemotherapy than patients with a normal lipid metabolism. Furthermore, mixed lipid metabolism disorders are more likely to cause severe liver damage.

\section{Abbreviations \\ ALT: Alanine aminotransferase; HDL-C: High-density lipid cholesterol; LDL- C: Low-density lipoprotein cholesterol; NAFLD: Non-alcoholic fatty liver disease; TC: Total cholesterol; TG: Triglycerides}

\section{Acknowledgements}

None.

\section{Funding}

The authors have no funding to disclose.

\section{Availability of data and materials}

The datasets analysed during the current study are available from the corresponding author upon reasonable request.

\section{Authors' contributions \\ $J L N$, YS, NZ and YLD conceived and designed the study. YS, JC, LJY, WY, DM and WKL assessed and diagnosed the patients. All authors were involved in the acquisition, analysis and interpretation of the data, with JLN taking the primary role in the statistical analysis. YS, NZ and YLD drafted the manuscript. All authors were involved in critically revising the manuscript and provided final approval of the manuscript.}

\section{Ethics approval and consent to participate}

The study was approved by the Medical Ethics Committee of Jingjiang People's Hospital. Written consent was obtained from all participants.

\section{Consent for publication}

Not applicable.

\section{Competing interests}

The authors declare that they have no competing interest.

\section{Publisher's Note}

Springer Nature remains neutral with regard to jurisdictional claims in published maps and institutional affiliations.

\section{Author details}

'Department of Oncology, JingJiang People's Hospital, the Seventh Affiliated Hospital of Yangzhou University, Jiangsu 214500, China. ${ }^{2}$ Department of Endocrinology, JingJiang People's Hospital, the Seventh Affiliated Hospital of Yangzhou University, Jiangsu 214500, China. ${ }^{3}$ Department of Neurology, Jing Jiang People's Hospital, the Seventh Affiliated Hospital of Yangzhou University, Jiangsu 214500, China. ${ }^{4}$ Department of Clinical Pharmacy, Jing Jiang People's Hospital, the Seventh Affiliated Hospital of Yangzhou University, No. 28, Zhongzhou Road, Jingjiang, Jiangsu 214500, China.

Received: 15 February 2019 Accepted: 29 April 2019

Published online: 10 May 2019

\section{References}

1. Feliu J, Heredia-Soto V, Girones R, Jimenez-Munarriz B, Saldana J, GuillenPonce C, Molina-Garrido MJ. Can we avoid the toxicity of chemotherapy in elderly cancer patients? Crit Rev Oncol Hematol. 2018;131:16-23.

2. Oun R, Moussa YE, Wheate NJ. The side effects of platinum-based chemotherapy drugs: a review for chemists. Dalton Trans. 2018;47:6645-53.

3. Pilgrim $\mathrm{CH}$, Thomson BN, Banting S, Phillips WA, Michael M. The developing clinical problem of chemotherapy-induced hepatic injury. ANZ J Surg. 2012; 82:23-9.

4. Leung J, Brady JL, Crook MA. The clinical importance of recognizing capecitabine-induced hypertriglyceridemia: a case report and review of the literature. J Clin Lipidol. 2018;12:1371-3.

5. Han GH, Huang JX. Hypertriglyceridemia and hyperglycemia induced by capecitabine: a report of two cases and review of the literature. J Oncol Pharm Pract. 2015;21:380-3.

6. Hossain MA, Amin A, Paul A, Qaisar H, Akula M, Amirpour A, Gor S, Giglio S, Cheng J, Mathew R, et al. Recognizing obesity in adult hospitalized patients: a retrospective cohort study assessing rates of documentation and prevalence of obesity. J Clin Med. 2018;7:203.

7. Lee SW, Lee TY, Yang SS, Tung CF, Yeh HZ, Chang CS. Risk factors and metabolic abnormality of patients with non-alcoholic fatty liver disease: either non-obese or obese Chinese population. Hepatobiliary Pancreat Dis Int. 2018;17:45-8.

8. Sweet PH, Khoo T, Nguyen S. Nonalcoholic fatty liver disease. Prim Care. 2017;44:599-607.

9. Najjar RS, Moore CE, Montgomery BD. A defined, plant-based diet utilized in an outpatient cardiovascular clinic effectively treats hypercholesterolemia and hypertension and reduces medications. Clin Cardiol. 2018;41:307-13.

10. Leoni S, Tovoli F, Napoli L, Serio I, Ferri S, Bolondi L. Current guidelines for the management of non-alcoholic fatty liver disease: a systematic review with comparative analysis. World J Gastroenterol. 2018;24:3361-73.

11. Wang G, Su C, Yin T. Paclitaxel and platinum-based chemotherapy results in transient dyslipidemia in cancer patients. Mol Clin Oncol. 2017;6:261-5.

12. Wang SC, D'Angelica MI. Chemotherapy-associated hepatotoxicity and hepatic resection for metastatic colorectal Cancer. Curr Color Cancer Rep. 2014;10:195-203.

13. Michie CO, Sakala M, Rivans I, Strachan MW, Clive S. The frequency and severity of capecitabine-induced hypertriglyceridaemia in routine clinical practice: a prospective study. Br J Cancer. 2010;103:617-21.

14. Duwe G, Knitter S, Pesthy S, Beierle AS, Bahra M, Schmelzle M, Schmuck RB, Lohneis P, Raschzok N, Ollinger R, et al. Hepatotoxicity following systemic therapy for colorectal liver metastases and the impact of chemotherapyassociated liver injury on outcomes after curative liver resection. Eur J Surg Oncol. 2017;43:1668-81.

15. Jarzabek MA, Proctor WR, Vogt J, Desai R, Dicker P, Cain G, Raja R, Brodbeck J, Stevens D, van der Stok EP, et al. Interrogation of transcriptomic changes associated with drug-induced hepatic sinusoidal dilatation in colorectal cancer. PLoS One. 2018;13:e0198099.

16. Shtriker MG, Peri I, Taieb E, Nyska A, Tirosh O, Madar Z. Galactomannan more than pectin exacerbates liver injury in mice fed with high-fat, highcholesterol diet. Mol Nutr Food Res. 2018;62:e1800331.

17. Zheng $Z, Y u$ H, Xiong $B$, Shen $S$, Yang H, Zhou Y. The incidence and risk factors of hepatotoxicity induced by perioperative hyperthermic 
intraperitoneal chemotherapy in gastrointestinal carcinoma patients: a retrospective study. Onco Targets Ther. 2018;11:5715-22.

18. Filliol A, Piquet-Pellorce C, Le Seyec J, Farooq M, Genet V, Lucas-Clerc C, Bertin J, Gough PJ, Dimanche-Boitrel MT, Vandenabeele P, et al. RIPK1

protects from TNF-alpha-mediated liver damage during hepatitis. Cell Death Dis. 2016;7:e2462.

19. Romestaing C, Piquet MA, Letexier D, Rey B, Mourier A, Servais S, Belouze M, Rouleau V, Dautresme M, Ollivier I, et al. Mitochondrial adaptations to steatohepatitis induced by a methionine- and choline-deficient diet. Am J Physiol Endocrinol Metab. 2008;294:E110-9.

20. Jara M, Bednarsch J, Malinowski M, Pratschke J, Stockmann M. Effects of oxaliplatin-based chemotherapy on liver function--an analysis of impact and functional recovery using the LiMAx test. Langenbeck's Arch Surg. 2016;401: 33-41.

21. Vincenzi B, Russo A, Terenzio A, Galvano A, Santini D, Vorini F, AntonelliIncalzi R, Vespasiani-Gentilucci U, Tonini G. The use of SAMe in chemotherapy-induced liver injury. Crit Rev Oncol Hematol. 2018;130:70-7.

Ready to submit your research? Choose BMC and benefit from:

- fast, convenient online submission

- thorough peer review by experienced researchers in your field

- rapid publication on acceptance

- support for research data, including large and complex data types

- gold Open Access which fosters wider collaboration and increased citations

- maximum visibility for your research: over $100 \mathrm{M}$ website views per year

At $\mathrm{BMC}$, research is always in progress.

Learn more biomedcentral.com/submissions 